\title{
DEVELOPMENT OF A "KERR EFFECT" DEVICE TO MEASURE PATIENTS URINE GLUCOSE LEVELS OF DIABETES MELLITUS (DM)
}

\author{
NurunNayiroh $^{1 *}$, Kusairi ${ }^{2}$ \\ ${ }^{1,2}$ Department of Physics, Science and Technology of Faculty, Maulana Malik Ibrahim Malang \\ Jl. Gajayana No. 50 Malang 65144
}

Recieved: $12^{\text {th }}$ November 2018; Revised: $12^{\text {th }}$ April 2019; Accepted: $30^{\text {th }}$ April 2019

\begin{abstract}
The development of the "Kerr Effect" device has been carried out to measure urine glucose levels of Diabetes Mellitus (DM) patients by applying an external electric field of two parallel plate capacitors. The purpose of this research was to design and construct a new "Kerr Effect" device to measure urine glucose levels of DM patients, to determine the effects of external electric fields and glucose concentrations on light polarization. The measurement of the polarization changes was done by emitting polarized light through a glucose standard solution sample and urine samples of DM patients. In this measurement, the sample was irradiated with an external electric field $(0 \mathrm{kV} / \mathrm{m}-80 \mathrm{kV} / \mathrm{m})$ of parallel plate capacitors, and the changes of light polarisation in the sample were observed. The obtained data of the measurement have been collected and plotted as a graph. The results showed that as the electric field increased the changes in polarization have a tendency to increase. In addition, the amount of glucose concentration in the urine also affected the magnitude of the change in the polarization rotational angle of the light. Therefore, the changes in the polarization rotational angle increase linearly due to the influence of the external electric field and the concentration of glucose in the urine.
\end{abstract}

Keywords: Kerr Effect; Glucose Levels; Urine; Diabetes Mellitus (DM).

\section{Introduction}

Light polarization in a medium is an optical property that very useful to determine the characteristic of the medium such as the structure and composition, and it has been used to develop optical measurements techniques. The use of polarized light to observe the optical properties of a medium is a fascinating field of research to be studied intensively; this allows in some circumstances to be used to determine the characteristics of a structure or composition of the medium under study. Some materials have naturally active optical properties such as sucrose $\left(\mathrm{C}_{12} \mathrm{H}_{22} \mathrm{O}_{11}\right)$ and glucose $\left(\mathrm{C}_{6} \mathrm{H}_{12} \mathrm{O}_{6}\right)$ which are dextrorotatory and the proteins are mostly levorotatory. ${ }^{1}$

There are some liquids that do not have active optical properties but can be observed for optical activity. This can be done by providing a high electric field about $1-10$
$\mathrm{kV} / \mathrm{m}$ to the liquid, resulting in changes in optical properties of the material including rotation of the plane-polarized light. ${ }^{2}$

Diabetes Mellitus (DM) is a metabolic disease characterized by chronic hyperglycemia. Hyperglycemia is indicated by a high concentration of glucose in the blood. Excessive glucose will be released by the body through urine, thus it can be detected through urine. Glucose is a type of monosaccharide sugar derived from carbohydrates. Glucose has a chemical structure $\mathrm{C}_{6} \mathrm{H}_{12} \mathrm{O}_{6}$ which rotates the direction of the polarization plane. ${ }^{3}$ The optical activity of $\mathrm{C}_{6} \mathrm{H}_{12} \mathrm{O}_{6}$ also has been examined by previous research; it showed that the increase in the concentration of $\mathrm{C}_{6} \mathrm{H}_{12} \mathrm{O}_{6}$ increases the rotary angle of polarized light. ${ }^{4}$

Light polarization is the event of changing the direction of light waves vibration randomly into one vibration direction. Light consists of perpendicular oscillation electric

*Corresponding author.

E-Mail: nayiroh@gmail.com 
and magnetic fields and it is a transverse wave which can be polarized. ${ }^{5}$

Isotropic crystalline and liquid materials may become anisotropic under the influence of a strong electric field. This phenomenon is known as Kerr Effect. ${ }^{6}$

There are two types of Kerr effect which are described below ${ }^{7}$ :

a) Electro-Optical Kerr Effect

The phenomenon when a strong electric field changes the refractive index of a medium.

b) Optical Kerr Effect

The phenomenon where the incident light creates an electric field and it changes the refractive index of a medium.

If the corresponding refractive index is $n_{a}$ and $n_{a}$, the difference in the optical path is formulated :

$$
\delta=l\left(n_{\alpha}-n_{a o}\right)
$$

Where $l$ is the optical path length, $n_{a o}$ is the initial refractive index and $n_{a}$ is the final refractive index. When a light beam with a wavelength $(\lambda)$ passes through a medium in a strong electric field, $E$, it will produce a change in the refractive index $(\Delta n)$ in the direction of the electric field. This change in refractive index is defined by the equation ${ }^{8}(\operatorname{Ref})$ :

$\Delta n=K E^{2} \lambda$

$K$ is a Kerr constant.

The electric field is a space around the source of an electric charge where other electric charges will experience the noncontact electric force.

Based on the optical response of glucose in a strong electric field on changing the light polarization rotary angle, a Kerr effect device could be developed to be an alternative technique to measure glucose level. Therefore, this research was conducted according to the Kerr effect to measure urine glucose levels in Diabetes Mellitus patients. The greater the level of sugar in the blood, the greater the rotational angle of the polarization axis. This indicates that the level of blood sugar is proportional to the rotational angle of the polarization axis ${ }^{9}$. In this research, we observed the influence of glucose concentrations on the light polarization angle. In addition, the effect of an electric field magnitude on the angle was also examined.

\section{Methods}

\section{Equipment and materials}

The equipment used in this research were He-Ne Laser $1 \mathrm{~mW}$ with a wavelength of 633 $\mathrm{nm}$; a photo element; a high voltage power supply; parallel plate capacitors; a polarisator; a voltage amplifier analyzer; a digital multimeter. A cuvette with size $12.5 \times 12.5 \mathrm{x}$ $45 \mathrm{~mm}$, connecting cables with length $1=750$ $\mathrm{mm}$; an Electro Field Meter (EFM), a beaker glass $50 \mathrm{ml}$; a spatula; a glass stirrer; a measuring cup $50 \mathrm{ml}$, a dropping pipette; analytical scales; and spray bottles. A Urine Gluco Protein meter was used to check glucose content in the urine.

The materials used in this research were urine samples of Diabetes Melitus patients with glucosaria among others positive $1(+)$, positive $2(++)$, positive $3(+++)$, positive 4 $(++++)$ and DM in pregnancy. The material used as a standard was a glucose solution with glucose concentration variations of $0.5 \%$; $1 \% ; 1.5 \% ; 2 \%, 2.5 \% ; 3 \% ; 3.5 \% ; 4 \% ; 5 \%$ and $6 \%$

\section{Method}

\section{Equipment Design}

The design of the glucose level meter equipment was arranged is shown in Figure 1.

1) He-Ne Laser

2) Polarisator

3) Analyzer serves to observe changes in the polarization angle of light after passing through a transparent material

4) Parallel Plate Capacitors equipped with EFM

5) Light Detector

6) Digital multimeter

7) High-voltage Power Supply

8) Sample Place

9) Connecting Cable 


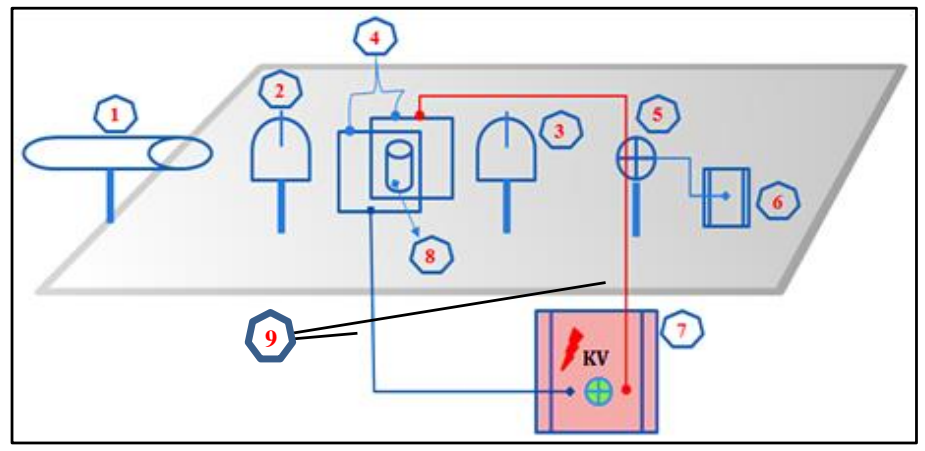

Figure 1. A glucose level meter scheme.

\section{Sample Preparation}

The urine samples of DM patients were taken from PROLANIS health facility of BPJS in Griya Bromo clinic, Bromo St..7A, Malang, East Java, Indonesia. To confirm the presence of sugar in the urine were confirmed using Urine Gluco Protein Test.

The standard solution with different concentration was made by dissolve $0.5 \mathrm{~g}$; $1 \mathrm{~g} ; 1.5 \mathrm{~g} ; 2 \mathrm{~g} ; 2.5 \mathrm{~g} ; 3 \mathrm{~g} ; 3.5 \mathrm{~g} ; 4 \mathrm{~g} ; 5 \mathrm{~g}$; and 6 $\mathrm{g}$ glucose in $100 \mathrm{ml}$ distilled water. The results are standard sample solutions with concentrations of $0.5 \% ; 1 \% ; 1.5 \% ; 2 \%$; $2.5 \% ; 3 \%, 3.5 \% ; 4 \% ; 5 \%$; and $6 \%$ glucose, respectively

\section{Calibration}

\section{- Background Polarization Intensity determination}

In this stage, a correction factor of the change in the polarization angle of the glucose level meter was obtained. The electric fields with different magnitudes ranging from 0 to $80 \mathrm{kV} \cdot \mathrm{m}^{-1}$ were applied to the glucose level meter with and without a container. In the electric field, the laser as an $0^{\circ}$ angle incident light was applied to the polarisator to obtain a polarized light. This polarized light then passed the container and the change in the angle from $0^{\circ}$ were detected with an analyzer and the minimum intensity value was taken as a background signal.

- Linearity response testing of the change in the polarization angle to the concentration of sugar.
In this stage, the linearity of polarization changes as a response to the increased sugar concentrations was observed. The linear response was conducted by irradiating sugar solution samples $(0.5 \% ; 1 \% ; 1.5 \% ; 2 \% ; 2.5 \% ; 3 \%, 3.5 \%$; $4 \%$; $5 \%$; and $6 \%$ ) without an external electric field $(E=0)$ and the external electric field is $10 \mathrm{kV} / \mathrm{m}$ with the direction of the laser beam $0^{\circ}$.

\section{Observation on the sample}

At this stage the measurement of polarization changes on the glucose solutions and DM patients' urine samples, in an external electric ranging from $0-80$ $\mathrm{kV} \cdot \mathrm{m}^{-1}$ was done. The $0^{\circ}$ polarized light obtained by polarisator were aimed at the sample with a different concentration in an external electric field. The changes in the angle of polarization were obtained using the analyzer then after correction factor, the polarization changes as the response functions of sugar and glucose concentrations were determined.

\section{Result and Discussion}

Table 1 shows the polarization change of the laser light as the response to the glucose standard solution with various concentrations and electric field magnitudes. From the Table, the polarization changes of the glucose standard solutions increase with increasing electric field magnitude. This linearity can be explained as the applied electric field was strong enough to change the optical property of the sample, where the electric field induced the rotation of polarization plane of sample. ${ }^{1}$ 
Data in Table 1 was used as a reference sample for measuring glucose levels in the DM patients' urine samples. Based on glucose concentration using Urine Gluco Protein Test, urine samples of DM patients in this research were divided into four types, namely: Positive
$1(+)$; positive $2(++)$;positive $3(+++)$, and positive $4(++++)$, based on glucose levels which are ranging from $0.5-1 \% ; 1-1.5 \% ; 2$ $-3.5 \%$; and $>3.5 \%$, respectively.

The sample test results are shown in Table 2.

Table 1. Changes in the at the rotary angle of glucose solution

\begin{tabular}{lllllllllll}
\hline \multirow{2}{*}{$\mathbf{E}$} & \multicolumn{10}{c}{ Glucose Concentration } \\
\cline { 2 - 7 }$/ \mathbf{m})$ & $\mathbf{0 . 5 \%}$ & $\mathbf{1 \%}$ & $\mathbf{1 . 5 \%}$ & $\mathbf{2 \%}$ & $\mathbf{2 . 5 \%}$ & $\mathbf{3 \%}$ & $\mathbf{3 . 5 \%}$ & $\mathbf{4 \%}$ & $\mathbf{5 \%}$ & $\mathbf{6 \%}$ \\
\hline 0 & 1.00 & 2.00 & 2.50 & 3.00 & 3.50 & 4.50 & 5.00 & 5.5 & 6.50 & 7.50 \\
9.82 & 1.50 & 2.10 & 2.51 & 3.20 & 3.52 & 4.51 & 5.51 & 5.55 & 6.60 & 7.60 \\
19.77 & 1.52 & 2.20 & 2.53 & 3.25 & 3.54 & 4.53 & 5.52 & 5.60 & 6.70 & 7.65 \\
29.88 & 1.54 & 2.29 & 2.54 & 3.30 & 3.58 & 4.54 & 5.53 & 5.64 & 6.80 & 7.72 \\
39.46 & 1.57 & 2.32 & 2.55 & 3.35 & 3.60 & 4.56 & 5.54 & 5.69 & 6.90 & 7.80 \\
49.23 & 1.59 & 2.39 & 2.57 & 3.38 & 3.64 & 4.57 & 5.55 & 5.75 & 7.00 & 7.85 \\
58.95 & 1.61 & 2.45 & 2.58 & 3.40 & 3.70 & 4.58 & 5.56 & 5.80 & 7.20 & 7.95 \\
69.21 & 1.64 & 2.47 & 2.59 & 3.42 & 3.76 & 4.59 & 5.57 & 5.85 & 7.30 & 8.00 \\
79.11 & 1.67 & 2.49 & 2.60 & 3.49 & 3.85 & 4.59 & 5.59 & 5.90 & 7.40 & 8.20 \\
\hline
\end{tabular}

Table 2. The changes in light polarization rotational angle in urine samples of DM patients

\begin{tabular}{cccccc}
\hline & \multicolumn{5}{c}{ Urine Sample } \\
\cline { 2 - 6 } $\mathbf{E}(\mathbf{k V} / \mathbf{m})$ & $\begin{array}{c}\text { Pregnancy } \\
(+++)\end{array}$ & + & ++ & +++ & ++++ \\
\hline 0 & 3.50 & 1.00 & 2.00 & 5.00 & 5.50 \\
9.82 & 3.51 & 1.50 & 2.10 & 5.51 & 5.52 \\
19.77 & 3.52 & 1.52 & 2.20 & 5.52 & 5.54 \\
29.88 & 3.53 & 1.54 & 2.25 & 5.53 & 5.56 \\
39.46 & 3.55 & 1.57 & 2.30 & 5.54 & 5.58 \\
49.23 & 3.56 & 1.59 & 2.35 & 5.55 & 5.59 \\
58.95 & 3.57 & 1.65 & 2.40 & 5.56 & 5.62 \\
69.21 & 3.58 & 1.70 & 2.50 & 5.57 & 5.65 \\
79.11 & 3.59 & 1.75 & 2.57 & 5.59 & 5.70 \\
Concentration & $2.5 \%$ & $0.5 \%$ & $1 \%$ & $3.5 \%$ & $4 \%$ \\
\hline
\end{tabular}

Table 2 shows the urine sample of pregnancy type $(+++)$ found a change in the polarization angle of 3.50 without external field treatment. Based on the test using the Urine Gluco Protein Test, urine samples of pregnant patients with DM showed positive three (+++), with blue and laboratory results showed that glucose levels were 275.34 $\mathrm{mg} / \mathrm{dl}$. If referring to Table 1 the change in polarization angle of 3.50 indicates a glucose concentration of $2.5 \%$. For $(+)$ shows the change in polarization angle 1.00 ( of glucose concentration $0.5 \%)$; (++) of 2.00 (glucose concentration of $1 \%$ ); (+++) of 5.00 (glucose concentration of $3.5 \%)$ and (++++) of 5.50 (glucose concentration of $4 \%$ ). From these 
data, it can be seen that there is a match between the measurements using the Kerr Effect and Urine Gluco Protein Test devices in measuring glucose levels in the urine (ref).

The data results obtained in Table 2 were processed in relationship graphs between changes in the light polarization rotational angle and the electric field (Figure 2), the figure also shows no significant increase in changes in the polarization of the +++ and ++++ samples and the pregnancy sample seems constant with the electric field magnitude. This is due to the possibility that when testing glucose molecules they are deposited on the base of the cuvette so that the polarization angle does not change significantly. And relationship graph between changes in light polarization rotation angle and changes in glucose concentration of DM urine samples without an external electric field $(\mathrm{E}=0)$ (Figure 3).

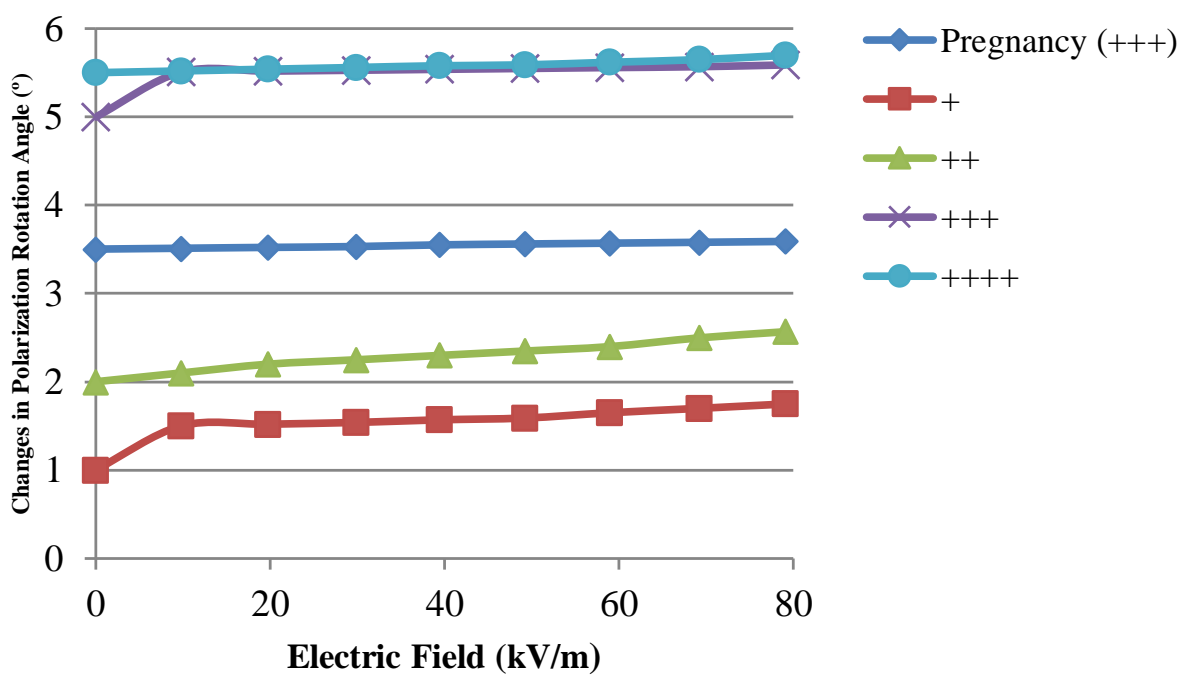

Figure 2. Graph of Changes in polarization rotation angle and electric field.

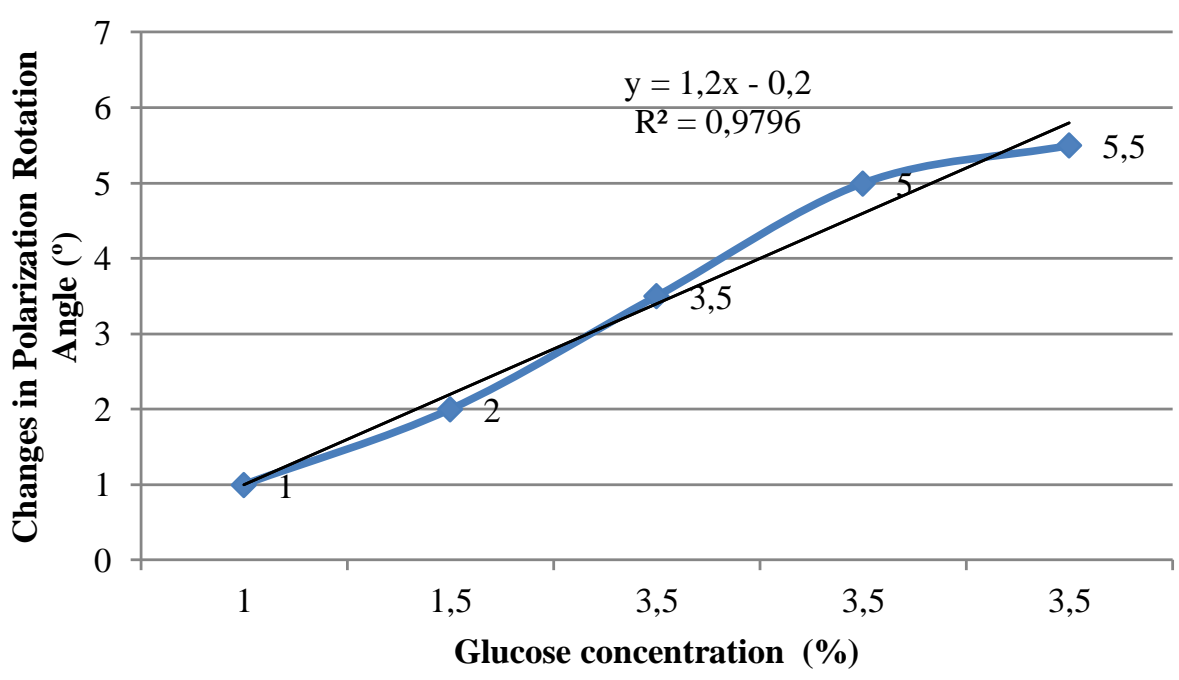

Figure 3. Graph of Changes in polarization rotation angle and glucose concentration in DM urine samples without electric field treatment.

Figure 2 shows that the change in light polarization rotational angle has increased with the increase in the electric field affecting the sample. Increasing changes in the polarization rotational angle of light due to the interaction between the external electric field and the polar molecule in the sample. The interaction between an electric field and a 
polar molecule will trigger the formation of a permanent electric dipole in a molecule. If the molecule is subjected to an external electric field, the electric dipole contained in the molecule will experience a direct rotation with the external electric field. The angle formed by the rotation of electric dipole in the molecule causes a change in the polarization rotation angle of light. The relationship between electric field interactions and electric dipoles causes changes of electric dipoles arrangement in the sample. These permanent dipoles will tend to be in line with the electric field. Electric dipoles in a material without the influence of an external electric field will be arranged randomly, while an electric dipole on a material affected by an external electric field will be arranged in the electric field direction ${ }^{10}$ (ref).

Changes in the polarization rotational angle of light are not only influenced by the electric field strength but also influenced by changes in glucose concentration of urine solution as shown in Figure 3. The results of data processing show changes in the light polarization rotational angle of DM urine sample increasing linearly as the glucose concentration changes in the urine sample. Where the higher the concentration of the solution, the greater of the rotation angle. The concentration of the medium passed by light affects the magnitude of rotation from the point of polarization. ${ }^{10}$

Glucose is one type of sugar that has active optical properties. Glucose can rotate the polarization plane in the right direction. Optical activity is the ability of certain substances to rotate the plane of light that is polarized in fields when light passes through crystals, liquids or solutions. ${ }^{11}$ The character of glucose in molecules forms with asymmetric central atoms is often also called as chiral molecules. The chiral molecule has properties similar to the lens where this molecule will respond and rotate the direction of the polarized light field. Another factor that causes changes in the polarization rotary angle of light is a laser beam. The electric field originating from a laser beam that interacts with glucose molecules in the DM urine will cause a change in the polarization rotation angle. ${ }^{1}$ Electric dipoles arranged regularly in a molecule due to an external electric field will experience a change in the light polarization rotation angle to left direction after being passed by a laser beam. It is due to electric dipole impact by the electric field from the polarized light emission. ${ }^{2}$

Based on the regression test in Figure 3 the results show that the system of this device follows the polarimeter law, which the higher glucose concentration in the urine is proportional to the change in polarization rotation angle with a correlation coefficient of $\mathrm{R}^{2}=0.9796$. It can be concluded that changes in glucose concentration in a solution are followed by changes in the polarization rotary angle. So this tool can be used to measure glucose levels in a solution that is in this research is urine fluid of DM patients.

\section{Conclusion}

Changes in the light polarization rotation angle have occurred due to external electric field influence and changes in glucose concentration of DM patients urine samples. The research results showed that the greater electric field strength of the urine sample, the greater polarization angle produced. The polarization rotary angle increases linearly with the correlation coefficient of $\mathrm{R}^{2}=$ 0.9796. This is due to the interaction of the external electric field and glucose molecules in the urine sample of DM patients.

\section{Acknowledgement}

to the research was supported by BOPTN research grant 2018 program from DIKTIS of Indonesia Republic Religion Ministry and LPPM of UIN Maulana Malik Ibrahim Malang.

\section{References}

1. Wibowo, H., EkoSugiyanto, K. Sofjan Firdausi, IndrasMarhaendrajaya. Effect of Medan Polarity on External Electricity and Polarization Angle of Laser Diodes for Kerr Effects Observation. Periodic Physics. 2006; 9 (1): 31-36. 
2. Sukarsono Kristantyo. Study of Kerr Effect to Purity of Aquades Test, PAM Water and Well Water, Periodic Physics. 2008; 11 (1): 4.

3. Habibana. Glucose. Brawijaya University of Malang.2014. Available from http://habibana.staff.ub.ac.id/files/2014/08 /GLUKOSA.pdf

4. Tjia.M.O. Wave. Bandung: ITB Press.1993.

5. Halliday, D., and Resnick, R. Physics $3^{\text {rd }}$ edition. Jakarta: Erlangga.1993.

6. Soedojo, Peter. 1992. Principles of Physics Volume III, Optics. Yogyakarta:Gadjah Mada University Press. In: KristantyoSukarsono, et all. Study of Kerr Effect to Purity of Aquades Test, PAM Water and Well Water. Periodic Physics. 2008 January; 11(1): 9-18.

7. Kristi, Julia, et al. The Effect Of Vco Quality (Virgin Coconut Oil) On Constant Value Of Characteristics and Changes In Polarization Angle Light. Proceedings of the Scientific Meeting and Presentation Basic Research of Nuclear Science and Technology. 2012 July: 28-34.
8. Officer Linda, et al. Effects of External Electric Fields on the of Polarization Rotary Angle of Laser Beams in Glycerin. Journal of Science \& Mathematics (JSM). 2007;15(2):79.

9. Lailia Nurafik, Muhammad, et al. Effect of Sugar Levels in Human Blood Against the Angle of Rotating Axes Polarization Using a Non-Invasive Polarmeter. Physics Departement FMIPA, Malang State University.Journal-online. 2019.

10. Supramono, Eddy, et al.. Basic Physics 2. Malang: UM Press.2005.

11. Sugiyarni, Anik. Determination of Glucose in Granulated Sugar Using the Faraday Effect Method. Surakarta:Department of Physics FMIPA SebelasMaret University.2010. 